\title{
Research on the Protection Countermeasures for Asymptomatic Patients in COVID-19
}

\author{
Yan-Yan Wu', Chang-Zheng Chen" ${ }^{1}$, Rong-Zhen Huang1, Guo-Qing Xu' ${ }^{1}$, Bin-Wen Xia ${ }^{2}$, Yang Yang ${ }^{3}$ \\ ${ }^{1}$ Qiantao Health Center, Guiyang, China \\ ${ }^{2}$ Guizhou University of Traditional Chinese Medicine, Guiyang, China \\ ${ }^{3}$ School of Nursing and Health, Henan University, Kaifeng, China \\ Email: 1798882655@qq.com
}

How to cite this paper: $\mathrm{Wu}, \mathrm{Y} .-\mathrm{Y} ., \mathrm{Chen}$, C.-Z., Huang, R.-Z., Xu, G.-Q., Xia, B.-W. and Yang, Y. (2021) Research on the Protection Countermeasures for Asymptomatic Patients in COVID-19. Open Access Library Journal, 8: e7250.

https://doi.org/10.4236/oalib.1107250

Received: February 17, 2021

Accepted: March 19, 2021

Published: March 22, 2021

Copyright $\odot 2021$ by author(s) and Open Access Library Inc.

This work is licensed under the Creative Commons Attribution International License (CC BY 4.0).

http://creativecommons.org/licenses/by/4.0/

(c) (i) Open Access

\begin{abstract}
Objective: To analyze the problems of protection COVID-19 asymptomatic infected patients, so as to ensure the safety of medical staff working in the epidemic and avoid the second outbreak. Methods: Through the analysis of main symptoms of the COVID-19 asymptomatic infected patients. Know the problems that if it is contagious, infectious strength, and the infection route etc. And take some measures such as launching medical observation for the close contacts of COVID-19 patients, investigating into epidemiology of cluster epidemic, active detection of exposed populations during the process of tracing the source of infection patients etc. to find asymptomatic infected patients. The prevention and control of asymptomatic infected persons should be strengthened by further improving the prevention and control program, increasing screening and monitoring, strengthening management and treatment, and strengthening mass prevention and control. Results: Through this method, COVID-19 asymptomatic infected patients can be detected as early as possible, and its effective prevention and control. Conclusion: The analysis of the protection problem of COVID-19 asymptomatic infector has important guiding significance to the protection of medical staff and individuals in the epidemic treatment.
\end{abstract}

\section{Subject Areas \\ Infectious Diseases}

\section{Keywords}

COVID-19, Asymptomatic Infected, Protection 


\section{Background}

In December 2019, COVID-19 broke out in Wuhan, Hubei, and then quickly spread to the world [1]. The National Health Commission has brought it into the category $\mathrm{B}$ management of legal infectious diseases, and has taken preventive and control measures for category A infectious diseases. On January 31, the World Health Organization (WHO) declared COVID-19 a public health emergency of international concern (PHEIC) [2] [3].

So far, although New Coronavirus pneumonia has been effectively controlled in China, it mainly blocks the spread of virus and has not yet developed effective vaccines. Patients with symptomatic infection of COVID-19 are easy to find, and effective measures such as isolation can be taken, while patients with asymptomatic infection of COVID-19 are difficult to find, which increases the difficulty of prevention and the risk of transmission. In March $26^{\text {th }}, 2020$, the Henan provincial health and Health Committee official informed that a Jiaxian People's Hospital doctor and two colleagues were dining at the same table in the hotel. One of the colleagues had a history of travel in Wuhan, and 14 days after their return to quarantine. In March $25^{\text {th }}, 2020$, the hospital conducted a physical examination, and found that it was asymptomatic [4] in the nucleic acid screening. In March 29 $9^{\text {th }}, 2020$, a New Coronavirus pneumonia infection in Henan. During the period of going to the countryside to sweep graves, the infected people were infected by eating three times with asymptomatic infected people [5]. At present, the prevention of asymptomatic infection is imminent, and effective measures will further reduce the risk of respread of the epidemic. In January 28, 2020, the national health and Health Committee issued the COVID-19 infection prevention and control plan (Third Edition), which included the asymptomatic infection of New Coronavirus in the prevention and control management. In the subsequent revision process, it made clear and specific requirements for the reporting and management of asymptomatic infected persons [6].

\section{Methods}

Asymptomatic infection of New Coronavirus pneumonia (referred to as asymptomatic infection) is characterized by no obvious clinical symptoms, such as fever, fatigue, cough, sore throat and other self-perceived or clinically recognizable symptoms, but nucleic acid detection or IgM antibody detection is positive [7]. Asymptomatic infection can be divided into two situations: one is that the infected person's nucleic acid or IgM antibody test is positive, after 14 days of incubation period observation, there are no self-perceived or clinically recognizable symptoms and characteristics, and they are always asymptomatic infection; the other is that the infected person's nucleic acid or IgM antibody test is positive, there are no self-perceived or clinically recognizable symptoms and signs at the time of sampling, but with the increase of time Later, some clinical manifestations appeared, that is, in the latent period of "asymptomatic infection" state.

According to the monitoring data of close contacts carried out by the state 
and some provinces, the close contacts of asymptomatic infected people have the second generation of cases. In epidemiological investigation, it is found that some asymptomatic infected people lead to the aggregated epidemic situation [8]. Small sample size studies show that the viral load in respiratory samples of asymptomatic infected people is not much different from that of confirmed cases [9]. According to the current monitoring and research, asymptomatic patients are infectious, but the length of infection period, infectious strength and transmission mode need further scientific research. Some experts believe that in view of the asymptomatic infection of respiratory tract specimens can detect pathogenic nucleic acid, but because there is no cough, sneezing and other clinical symptoms, the chance of transmission caused by pathogen excretion is relatively less than the confirmed cases.

Therefore, it is necessary to do a good job in asymptomatic infection monitoring, increase the screening efforts, and expand the scope of detection to the close contacts, key areas and key groups of the found cases and asymptomatic infection. Once asymptomatic infection is found, it is necessary to carry out strict isolation and medical observation according to the "four early" requirements, and implement isolation medical observation for close contacts.

First of all, because the transmission of asymptomatic infection is hidden, it has no obvious symptoms and signs, which is difficult to find in the crowd, and the resulting transmission is difficult to prevent.

Secondly, because asymptomatic pneumonia patients have subjective symptoms, the patients with mild or atypical symptoms may think that they are not infected with new coronavirus and do not take the initiative to go to medical institutions, which is difficult to be found in daily diagnosis and treatment.

Third, there are limitations. Due to the existence of detection window period, it is difficult to find all asymptomatic infected persons by nucleic acid detection and serological detection methods. The existing asymptomatic infected persons are mainly found by active screening of close contacts of cases, investigation of infection sources, investigation of aggregated epidemic situation and active detection of personnel in high-risk areas, and some asymptomatic infected persons are difficult to be found.

Therefore, it is found that asymptomatic infections, the first is the medical observation of the close contacts of New Coronavirus pneumonia cases; the second is the epidemiological investigation of the cluster epidemic situation; the third is the active detection of the exposed population in the process of tracing the infection source of the New Coronavirus pneumonia cases; fourth, the part of the brigade that has some cases of domestic and foreign New Coronavirus pneumonia cases continuing to spread. Active detection was carried out by people with travel history and residence history.

Because some asymptomatic infected people are always asymptomatic, it is impossible to find and isolate asymptomatic infected people as the leading measure in the actual prevention and control work. Therefore, we should con- 
tinue to focus on timely detection, isolation and diagnosis of patients, and do a good job in close contact management. China's experience shows that timely detection and isolation of confirmed cases, and appropriate measures to reduce interpersonal contact can basically block the spread of the epidemic.

\section{Results}

\subsection{Further Improve the Control Program}

Through sampling a certain proportion of samples in key epidemic areas, conducting investigation and epidemiological analysis of asymptomatic infected persons, improving prevention and control measures, revising and improving prevention and control programs and diagnosis and treatment programs, scientifically coping with the infection risk of asymptomatic infected persons, and curbing the possible formation of a new round of epidemic transmission.

\subsection{Increase Screening and Monitoring.}

Targeted efforts should be made to increase the screening, and the scope of detection should be extended to the close contacts of cases and asymptomatic infected persons, key areas and key groups. Combined with the actual situation of returning to work, production and school, we should strengthen the monitoring of key cities, key people and key places to find hidden dangers to the greatest extent. Do a good job in the prevention of cross-border import and export of epidemic situation, and conduct nucleic acid testing for all entry personnel. After the asymptomatic infection was found, the epidemiological investigation was carried out in time to find out the source and release the information openly and transparently.

\subsection{Strengthen Management and Treatment}

Once asymptomatic patients are found, they should be strictly isolated and managed according to the "four early" requirements, and corresponding isolation medical observation should be carried out for close contacts. If there are symptoms during isolation, they should be immediately transferred to designated medical institutions for treatment.

\subsection{Strengthen Group Prevention and Control}

Adhere to the combination of group and specialty, strengthen the popularization of epidemic prevention knowledge, guide the public in scientific protection, carry out extensive training, and improve the prevention and control ability and level of grassroots disease control personnel, medical personnel and community workers.

\subsection{How to Do Well in Personal Protection}

While strengthening the awareness of self-protection and health, the public should also strengthen environmental hygiene and personal hygiene protection, 
and develop habits such as washing hands, cleaning and disinfection, wearing masks scientifically, and serving of individual dishes. We should carry out the Patriotic Health Campaign extensively, spread health knowledge to every family and individual, form good health habits and civilized, healthy and green lifestyle, and improve the level of mental health and health literacy. If the individual nucleic acid or IgM antibody test is positive, there is no need to panic. We should take the initiative to cooperate with the medical and health institutions to do a good job in health monitoring and isolation medical observation, report in time when fever, cough and other symptoms appear, and accept the standardized diagnosis and treatment of medical institutions.

By improving the prevention and treatment plan, increasing screening and monitoring, strengthening management and treatment, and strengthening group prevention and group control, etc., actively dig out hidden asymptomatic infections to prevent further transmission, achieved the purpose of prevention and control of asymptomatic infections.

\section{Conclusion}

At present, the epidemic of New Coronavirus pneumonia is spreading rapidly all over the world. With the joint efforts of the whole nation, the epidemic situation has been temporarily controlled. However, there are still a large number of asymptomatic patients. Under the premise of understanding the characteristics of asymptomatic patients, we should further improve the prevention and control plan, and intensify targeted screening and monitoring to find the greatest hidden danger. At the same time, individuals should also take protective measures, develop good health habits, and try to avoid going to the places where people gather, so as to avoid the second outbreak of the epidemic.

\section{Author's Contributions}

WU Yan-yan Conceived and conducted the study.

CHEN Chang-zheng, HUANG Rong-zhen, XU Guo-qing, XIA Bin-wen, Yang-yang Provided overall supervision of the work and critical revision of the paper, and contributed to the conception and conduction of the study.

\section{Conflicts of Interest}

The authors certify that they have no conflict of interest with respect to the research, authorship, and/or publication of this article.

\section{References}

[1] Zhu, Y.C., Tan, L., Liu, L., et al. (2020) Comparative Analysis of the Characteristics and Drug Treatment of New Coronavirus Pneumonia and SARS. Journal of Clinical Drug Treatment, 18, 15-23.

[2] Liu, L., Zhang, J.D., Ma, S.S., et al. (2020) Qualitative research on Frontline Nurses' COVID-19 Care Experience. Journal of Qilu Nursing, 26, 3-6.

[3] She, J.T. and Liu, W.J. (2020) Epidemiological Characteristics and Prevention and 
Control Measures of New Coronavirus Pneumonia in Children. Journal of Tropical Medicine, 20, 153-155+172.

[4] Beijing Youth Daily (2020) Novel Coronavirus Pneumonia Confirmed in Henan. http://epaper.ynet.com/html/2020-03/30/content_350745.htm?div=-1

[5] Henan Health Committee (2020). http://wsjkw.henan.gov.cn/2020/03-29/1315266.html

[6] National Health and Health Committee (2020) Notice of the National Health and Health Commission's Office on the Prevention and Control of New Coronavirus Infection (Third Edition).

http://www.nhc.gov.cn/jkj/s7923/202001/470b128513fe46f086d79667db9f76a5.shtml

[7] Kang, Z.F., Zhou, Y.Q., Zhang, L.X., et al. (2020) New Coronavirus Infection Eye Protection and Integrated Traditional Chinese and Western Medicine Clinical Prevention and Control Suggestions. China Ophthalmology, 30, 75-79.

[8] Qiao, D. (2020) Nine Questions about “Asymptomatic Infected Persons” Experts Have the Most Complete Interpretation. Science Grand View Garden, No. 8, 54-59.

[9] Gao, X., Chen, W., Guo, L., Zhang, T.Z. and Zhao, H.Q. (2020) Asymptomatic Infection of New Coronavirus Pneumonia and Its Challenges to Epidemic Prevention and Control. Chinese Journal of Epidemiology, 41, 1985-1988. 